\title{
Diffusional anomaly and network dynamics in liquid silica
}

\author{
Ruchi Sharma, Anirban Mudi and Charusita Chakravarty* \\ Department of Chemistry, \\ Indian Institute of Technology-Delhi, \\ New Delhi: 110016, India.
}

\begin{abstract}
The present study applies the power spectral analysis technique to understand the diffusional anomaly in liquid silica, modeled using the BKS potential. Molecular dynamics simulations have been carried out to show that power spectrum of tagged particle potential energy of silica shows a regime with $1 / f^{\alpha}$ dependence on frequency $f$ which is the characteristic signature of multiple time-scale behaviour in networks. As demonstrated earlier in the case of water (J. Chem. Phys., 122, 104507 (2005)), the variations in the mobility associated with the diffusional anomaly are mirrored in the scaling exponent $\alpha$ associated with this multiple time-scale behaviour. Our results indicate that in the anomalous regime, as the local tetrahedral order decreases with temperature or pressure, the coupling of local modes to network reorganisations increases and so does the diffusivity. This symmetry-dependence of the vibrational couplings is responsible for the connection between the structural and diffusional anomalies.
\end{abstract}

* Author for correspondence (Tel: (+) 9111 26591510; Fax: (+) 91112686 2122; E-mail: charus@chemistry.iitd.ernet.in) 


\section{INTRODUCTION}

Tetrahedral network-forming liquids are characterised by strong, local anisotropic interactions which impose a local tetrahedral order with four nearest neighbours, in contrast to the local icosahedral order characteristic of the random closed-packing structures seen in simple liquids. The interactions in simple liquids are dominated by strong, short-range repulsions and weak isotropic attractions; typical examples are the rare gases and liquid metals ${ }^{1}$. The best known example of a tetrahedral liquid is water where each oxygen atom can form four tetrahedrally disposed hydrogen bonds with its neighbours. At room temperature, the hydrogen bond energy is of the order of $5-10 k_{B} T$ and the hydrogen bonds in water can break and reform on timescales of the order of picoseconds resulting in a fluctuating, random three-dimensional network of water molecules 2.3 . A second important example is liquid silica where the $\mathrm{SiO}_{4}$ tetrahedra are linked to form a random network ${ }^{4.5}$. Interactions in $\mathrm{SiO}_{2}$ have a mixed ionic-covalent character with $\mathrm{Si}-\mathrm{O}$ bond energy of approximately 450 $\mathrm{kJ} \mathrm{mol}^{-1}$ such that in the temperature regime above $2000 \mathrm{~K}$ where the liquid phase is stable, it is of the order of $25 k_{B} T$ or less ${ }^{6}$. The liquid phases of Si, Ge, Sb, Bi and Ga are expected to be tetrahedral as are those of several ionic compounds such as $\mathrm{BeF}_{2}$ and intermetallics such as InSb, GaAs, GaP, HgTe, CdTe and $\mathrm{CdSe}^{7}$.

The liquid state thermodynamic and kinetic properties of tetrahedral liquids are in many respects anomalous when compared with those of simple liquids 2.5 .8 . The best known of the thermodynamic anomalies is the density anomaly shown by the existence of a temperature of maximum density (TMD). Unlike simple liquids where density decreases monotonically with temperature, in tetrahedral liquids, increasing thermal kinetic energy over certain density regimes results in progressive destruction of the network, consequent compaction and a resulting increase in density. At atmospheric pressure, the TMD of water occurs at 279K whereas that of silica occurs at approximately 5000K. The behaviour of response functions of tetrahedral liquids, such as the isothermal compressibility $(\kappa)$ and the isobaric heat capacity $\left(C_{p}\right)$, can also be anomalous. In simple liquids, both $C_{p}$ and $\kappa$ decrease monotonically with decreasing temperature but, for tetrahedral liquids, both $\kappa$ and $C_{p}$ as a function of temperature along an isobar can show minima. Thermodynamic arguments can be used to show that the existence of a density anomaly implies the existence of compressibility and heat capacity anomalies ${ }^{2}$. In addition to the thermodynamic anomalies, tetrahedral liquids also show kinetic anomalies. For example, the diffusional anomaly corresponds to a regime in which the diffusivity increases as a function of density, in contrast to simple liquids 
where diffusivity decreases with density due to increasing steric hindrance. Qualitatively, this anomalous behaviour of the mobility can be understood in terms of the disruption of the tetrahedral network by increasing pressure or density which facilitates translational motion of the water molecules. The phase diagram of tetrahedral liquids can show a number of unusual features, such as a negatively sloped melting line on the pressure-temperature phase diagram. There is also increasing evidence that tetrahedral liquids can show polyamorphism i.e. the existence of low and high-density liquid or glassy phases.

Over the past decade, a better understanding of the connection between the various anomalous properties of tetrahedral liquids has been obtained from a combination of experimental and simulation studies on water, and to a lesser extent, silica. A key innovation has been the introduction of order metrics to quantify the nature as well as the extent of structural order present in such liquids $\frac{910}{}$. The extent of local tetrahedral order present around a tagged tetrahedral centre (e.g.O atom in water and $\mathrm{Si}$ atom in $\mathrm{SiO}_{2}$ ) is gauged by a tetrahedral order parameter $q$ defined as

$$
q=1-\frac{3}{8} \sum_{j=1}^{3} \sum_{k=j+1}^{4}\left(\cos \psi_{j k}+1 / 3\right)^{2}
$$

where $\psi_{j k}$ is the angle between the bond vectors $\mathbf{r}_{i j}$ and $\mathbf{r}_{i k}$ where $j$ and $k$ label the four nearest neighbour atoms of the same type e.g. the $q_{O}$ order parameter in water is defined using the positions of the four nearest oxygen atoms ${ }^{9.11}$. The translational order parameter, $\tau$, was defined as

$$
\tau=\left(1 / \xi_{c}\right) \int_{0}^{\xi_{c}}(g(\xi)-1) d \xi
$$

where $\xi=r \rho^{1 / 3}$ and $\xi_{c}=2.83^{12}$. The translational order parameter, as defined above, will capture the extent of pair correlations present in the liquid and can be thought of as a type of density-ordering, since $\tau$ will increase as the random close-packing limit is approached. The structure and dynamics of tetrahedral liquids can be understood in terms of an interplay between short-range tetrahedral ordering and translational or density ordering which promotes local icosahedral order seen in simple liquids. At a given temperature, $q$ will show a maximum and $\tau$ will show a minimum as a function of density; the loci of these extrema in the order define a structurally anomalous region in the density-temperature $(\rho T)$ plane where the tetrahedral and translational order parameters are found to be strongly correlated. The region of the density anomaly, where $\partial \rho / \partial T>0$, is bounded by the structurally anomalous region. The diffusionally anomalous region $(\partial D / \partial \rho>0)$ encloses the boundaries of the structurally anomalous region in the case of silica. In water, on the other hand, the 
boundaries of the diffusionally anomalous region lie between those of the structural and density anomalies. Thus one can think of tetrahedral liquids as displaying a cascade of anomalies where structural, diffusional and density anomalies occur consecutively as the degree of tetrahedral order is increased. Our understanding of the molecular level processes which give rise to this cascade effect is still incomplete. An important step in this direction is the demonstration of the cooperative origin of low-density domains in water which suggests that the probability density of centres of high local tetrahedral order must reach a certain threshold value before cooperative effects can precipitate the formation of ramified, low density clusters of water molecules ${ }^{11,13}$.

In a series of recent papers, we showed that power spectral analysis could be used to understand the connections between the local order, hydrogen bond network dynamics and the kinetic anomalies in water $\frac{14.15 .16 .17 .18 .19}{}$. The power spectral density of an observable $A(t)$ as a function of time $t$ is defined as follows ${ }^{20}$ :

$$
S(f)=\lim _{T \rightarrow \infty} \frac{1}{T}\left|\int_{-T / 2}^{T / 2} A(t) e^{2 \pi i f t} d t\right|^{2} .
$$

The power spectrum contains the same information as the time correlation function of the observable but it highlights its behaviour over a range of frequencies. The multiple time-scale dynamics of networks is reflected in the power spectrum, $S(f)$, as a $1 / f^{\alpha}$ dependence 21,22 . In the case of water, where the network is formed by the coupling of individual molecules by hydrogen bonds, the observables which are sensitive to local motions as well as network re-organisations were found to be the local tetrahedral order parameter and the tagged molecule potential energy. Fluctuations in these observables generated power spectra with three key features: (a) a local vibrational peak corresponding to the librational modes in water; (b) a multiple time scale region with $1 / f^{\alpha}$ behaviour and (c) a crossover to white noise at lower frequencies identifying the time scales for decay of correlations in the network. The diffusivity was shown to be strongly correlated with the exponent $\alpha$ of the $1 / f^{\alpha}$ region of the power spectra of the tagged particle potential energy fluctuations. Thus the power spectrum has been shown to provide a direct dynamical measure in the scaling exponent $\alpha$ of the degree of coupling of the librational modes to the network vibrations which complements the static structural measures, such as the coordination number distributions, used in earlier studies. Destruction of tetrahedral local order by temperature, pressure or ionic solutes facilitates the coupling of local, librational modes to the network motions and therefore the parallel behaviour of the structural and diffusional anomalies is not surprising. The power 
spectral analysis shows that the boundaries of the two regions cannot be identical because the structurally anomalous region is defined by the average values of the local order parameters whereas the diffusionally anomalous region is determined by the dynamical correlations in the fluctuations in the local order and energy.

In this paper, we apply the power spectral analysis techniques described above to understand the diffusional anomaly in liquid silica, modeled using the BKS potential23.24. While the behaviour of liquid silica and water has been shown to be parallel in many respects, there are some important differences $\frac{8,10,25,26,27,28}{}$. Among all the crystalline polymorphs of silica, only $\beta$-cristobalite has a melting line with a slightly negative slope having a change in volume equal to $-1 \%$ as compared to $-8.9 \%$ seen for water ${ }^{6}$. From the chemical bonding perspective, silica is thought of as an ionic liquid with strong covalent character. The tetrahedral motif in water is displayed by the Walrafen-pentamer structure, while in silica and many silicates, the $\mathrm{SiO}_{4}$ tetrahedra are the crucial structural unit. The O-H..O hydrogen bond in water is nearly linear while in silica, the Si-O-Si bond angle varies between 140$155^{\circ} \mathrm{C}^{29}$. It is therefore interesting to consider the extent to which the dynamical behaviour of the two networks is similar. Based on our studies of water, we have found the tagged particle (atom or molecule) energies as a convenient dynamical variable which is sensitive to local order and energy of the molecular environment. In addition, since it is defined as the interaction of a tagged atom or molecule with all other particles in the simulation cell, it is reasonably sensitive to network reorganisations over a fairly wide range of length and time scales. Therefore we focus on the tagged ion potential energies as the important local variable in a tetrahedral liquid and study the corresponding static distributions as well as the power spectrum of the fluctuations.

The paper is organised as follows. Section 2 discusses the computation of the tagged ion potential energies when Ewald summation is used to evaluate the long range, Coulombic interactions. Section 3 summarises the aspects of power spectral analysis required in this work. Computational details regarding the potential model and molecular dynamics simulations is given in Section 4. Section 5 and 6 contain the results and conclusions respectively. 


\section{COMPUTATION OF TAGGED MOLECULE POTENTIAL ENERGIES}

Parametric potentials for silica are typically constructed as a sum of long-range, Coulombic interactions, $U_{\text {coul }}$, and short-range van der Waals interactions, $U_{v d w}$, such that

$$
U=U_{c o u l}+U_{v d w}
$$

The Coulombic contribution comes from the charge-carrying $\mathrm{Si}$ and $\mathrm{O}$ atoms. We assume that the short-range interactions include only two-body terms, but if three-body terms are present the computation can be adapted. The functional form of the short-range potential used in this work is discussed in section 4.1. For a pair additive system, if $u_{S i}$ and $u_{O}$ are the tagged atom energies and $n_{S i}$ and $n_{O}$ are the number of $\mathrm{Si}$ and $\mathrm{O}$ atoms respectively, then the corresponding ensemble averages are related to the average configurational energy as: $2\langle U\rangle / N=\left(n_{S i} / N\right)\left\langle u_{S i}\right\rangle+\left(n_{O} / N\right)\left\langle u_{O}\right\rangle$.

The short range van der Waals interactions contribute an amount $\left(u_{i}^{v d w}\right)$ to the tagged atom potential energy $u_{i}$. Since the interactions are short-ranged, the minimum image convention can be applied and $u_{i}^{v d w}$ can be evaluated by summing over all pair interaction contributions between atom $i$ and all other atoms $j$ located in the central simulation cell. If the short-range potential has a finite value at the spherical cut-off radius, a densitydependent long-range correction term must be included in $u_{i}^{v d w}$.

To evaluate the Coulombic contribution to the tagged molecule potential energy, one must consider the Ewald summation technique for evaluating long-range forces ${ }^{30,31}$. The charge density distribution for a collection of $N$ point charges located in the central simulation cell is given by:

$$
\rho(\mathbf{r})=\sum_{i}^{N} q_{i} \delta\left(\mathbf{r}-\mathbf{r}_{i}\right)
$$

Note that $\mathbf{r}$ is a 3 -dimensional position vector and $\mathbf{r}_{i}$ is the position of the $i^{\text {th }}$ charge $q_{i}$ and that the central simulation cell is set up to be electrically neutral. The simulation cell is taken as cubic with edge length $L$. The Coulombic interaction energy between all charges in the central simulation box and all other periodic images is given by:

$$
U_{\text {coul }}=\frac{1}{2} \sum_{\mathbf{n}=0}^{\infty} \sum_{i=1}^{N} \sum_{j=1}^{N} \frac{q_{i} q_{j}}{4 \pi \epsilon_{0}\left|\mathbf{r}_{i j}+\mathbf{n}\right|}
$$

where $\mathbf{r}_{i j}$ is the vector distance between charges $q_{i}$ and $q_{j}, \mathbf{n}=\left(n_{x} L, n_{y} L, n_{z} L\right)$ denotes the set of possible translations of the simulation cell. The central simulation box corresponds to the $\mathbf{n}=0$ case and the prime on the first summation indicates that the series does not 
include the interaction $i=j$ for $\mathbf{n}=0$. Unlike in the case of the short-range van der Waals interactions, the inverse distance dependence of the Coulombic interaction implies that the summation cannot be restricted to just the central simulation cell and the above series will converge very slowly. Ewald summation replaces the slowly convergent series in equation (5) by a sum of two rapidly convergent series obtained by replacing the charge distribution $\rho(\mathbf{r})$ by a sum of two charge distributions, $\rho_{\text {screen }}$ and $\rho_{\text {gauss }}$. To obtain $\rho_{\text {screen }}(\mathbf{r})$, each point charge $q_{i}$ is surrounded by a neutralizing charge distribution, usually a Gaussian distribution centred at the location of $q_{i}$. To ensure that $\rho(\mathbf{r})=\rho_{\text {screen }}(\mathbf{r})+\rho_{\text {gauss }}(\mathbf{r}), \rho_{\text {gauss }}(\mathbf{r})$ must consist of Gaussian charges which neutralise the screening charges used to construct $\rho_{\text {screen }}$.

The potential energy contribution due to the screened charges is denoted by $U_{\text {screen }}$. The advantage of choosing neutralising Gaussian distributions of the form:

$$
\rho_{i}(\mathbf{r})=\frac{q_{i} \alpha^{3}}{\pi^{3 / 2}} \exp \left(-\alpha^{2} r^{2}\right)
$$

is that $U_{\text {screen }}$ can be readily evaluated using the complementary error function, er $f c$, as follows:

$$
U_{\text {screen }}=\frac{1}{2} \sum_{i=1}^{N} \sum_{j=1}^{N} \sum_{|\mathbf{n}|=\mathbf{0}}^{\infty}{ }^{\prime} \frac{q_{i} q_{j}}{4 \pi \epsilon_{0}} \frac{\operatorname{erfc}\left(\alpha\left|\mathbf{r}_{i j}+\mathbf{n}\right|\right)}{\left|r_{i j}+\mathbf{n}\right|}
$$

This contribution is frequently referred to as 'real space' summation and its rate of convergence depends upon the parameter $\alpha$ of the neutralising Gaussians. The larger the value of $\alpha$, the narrower the gaussian distribution and the shorter the range of interaction of the screened charges. Typically, $\alpha$ is chosen to ensure that the minimum image convention can be applied i.e. the range of the interactions is shorter than half the length of the simulation cell i.e. only the $|\mathbf{n}|=0$ case needs to be considered. One can now rewrite:

$$
\begin{aligned}
U_{\text {screen }} & =\frac{1}{2} \sum_{i=1}^{N} \sum_{j=1}^{N} \frac{q_{i} q_{j}}{4 \pi \epsilon_{0}} \frac{\operatorname{erfc}\left(\alpha\left|\mathbf{r}_{i j}\right|\right)}{\left|r_{i j}\right|} \\
& =\frac{1}{2} \sum_{i}^{N} u_{i}^{\text {screen }}
\end{aligned}
$$

where $u_{i}^{\text {screen }}$ represents the contribution from the screened charges to the tagged atom Coulombic energy of atom $i$.

The contribution of the Gaussian charge distribution, $\rho_{\text {gauss }}(\mathbf{r})$, to the Coulombic interaction energy can be evaluated very simply in reciprocal or $k$-space and is denoted by $U_{\text {rec }}$. The electrostatic potential, $\Phi_{\text {gauss }}$, associated with this charge distribution can be evaluated in real space using:

$$
-\nabla^{2} \Phi_{\text {gauss }}(\mathbf{r})=4 \pi \rho_{\text {gauss }}(\mathbf{r})
$$


or in the Fourier form in reciprocal space as:

$$
k^{2} \Phi_{\text {gauss }}(\mathbf{k})=4 \pi \rho_{\text {gauss }}(\mathbf{k})
$$

where $\rho_{\text {gauss }}(\mathbf{k})$ is the Fourier transform of $\rho(\mathbf{r})$. Fourier transforms of arrays of Gaussians can be computed analytically and one can then write $e^{31}$

$$
U_{\text {rec }}=\frac{1}{2} \sum_{i} q_{i} \Phi_{\text {gauss }}(\mathbf{r})
$$

Taking explicit summations over the partial charges, indexed by $j$, and the reciprocal lattice vectors, indexed by $|\mathbf{n}|$ one can write

$$
U_{\text {rec }}=\frac{1}{2 V_{0} \epsilon_{0}} \sum_{|n| \neq 0}^{\infty} \frac{\exp \left(-k^{2} / 4 \alpha^{2}\right)}{k^{2}}\left|\sum_{j}^{N} q_{j} \exp (-i \vec{k} \cdot \vec{r})\right|^{2}
$$

Using the above expression, $U_{\text {rec }}$ can be calculated using a double loop over $|\mathbf{n}|$ and $j$. Computing the tagged atom contribution, requires the modulus squared term to be replaced by a double sum over $i$ and $j$, where $i$ and $j$ label the partial charges. Rearranging the above expression

$$
\begin{aligned}
U_{\text {rec }} & =\frac{1}{2 V_{0} \epsilon} \sum_{j} \sum_{k \neq 0} \frac{q_{j}}{k^{2}} \exp \left(i \vec{k} \cdot \vec{r}_{j}-k^{2} / 4 \alpha^{2}\right) \sum_{i=0}^{N} q_{i} \exp \left(-i \vec{k} \cdot \overrightarrow{r_{n}}\right) \\
& =\frac{1}{2 V_{0} \epsilon} \sum_{j} \sum_{k \neq 0} \frac{q_{j}}{k^{2}} \exp \left(-k^{2} / 4 \alpha^{2}\right) \sum_{i=0}^{N} q_{i} \exp \left(-i \vec{k}\left(\overrightarrow{r_{j}}-\overrightarrow{r_{i}}\right)\right. \\
& =\frac{1}{2} \sum_{j} u_{j}^{r e c}
\end{aligned}
$$

Since the $u_{j}^{r e c}$ contribution multiplied by $(-i \vec{k})$ gives the force acting on charged site $j$, the Ewald summation subroutines supplied with the DL_POLY program require only a small modification in order to extract $u_{j}^{r e c 32.33}$.

It should be noted that the above expression for $U_{\text {rec }}$ includes a self-interaction term for the interaction of each Gaussian charge distribution with itself. When computing the Ewald summation to evaluate $U_{\text {coul }}$, it is necessary to remove these redundant self-interaction contributions, $U_{\text {self }}$, which are given by

$$
U_{\text {self }}=\sum_{j=1}^{N} u_{j}^{\text {self }}=(1 / 4 \pi \epsilon) \sum_{j} \frac{q_{j}^{2} \alpha}{\sqrt{\pi}}
$$

The complete expression for $U_{\text {coul }}$ will be

$$
U_{\text {coul }}=U_{\text {rec }}+U_{\text {screen }}-U_{\text {self }}
$$


The contributions to the tagged potential energy that comes from the electrostatic interactions is given by $\left(u_{i}^{r e c}+u_{i}^{\text {screen }}-u_{i}^{\text {self }}\right)$. The overall tagged molecule potential energy, $u_{i}$, of molecule $i$ will therefore be given by :

$$
u_{i}=u_{i}^{v d w}+u_{i}^{r e c}+u_{i}^{s c r e e n}-u_{i}^{\text {self }}
$$

\section{POWER SPECTRAL ANALYSIS}

The power spectral density of an observable $A(t)$ as a function of time $t$, has been defined in equation (3). In this work, the dynamical observables are the tagged atom potential energies, $u_{i}(t)$, where $i$ corresponds to a $\mathrm{Si}$ or $\mathrm{O}$ atom. Since we sample the potential energy signal at discrete times, the definition of the power spectral density must be changed to allow for discrete sampling 20 :

$$
S_{k}=S\left(f_{k}\right)=\frac{\left|F_{k}\right|^{2}}{N^{2}}
$$

where $N$ is the number of samples, and $F_{k}$ is the Discrete Fourier Transform (DFT) evaluated at the $k$-th frequency, i.e.,

$$
F_{k}=F\left(f_{k}\right)=\sum_{n=0}^{N-1} A_{n} e^{2 \pi i n k / N}
$$

(here we use the same normalization as ref. 20, the $k$-th frequency is $f_{k}=k /(N \Delta t), \Delta t$ is the sampling interval, and $k=0, \ldots, N-1)$. The dynamical time scales present in the system will clearly determine the behaviour of the power spectrum. Consider a simple situation in which there is a single relaxation frequency $\lambda$ in the system. If several such relaxation events take place at an average rate $n$ at times $t_{k}, k=1,2, \ldots$, then each such event will give rise to an exponential decay in some appropriate dynamical variable of the form $A_{0} \exp \left[-\lambda\left(t-t_{k}\right)\right]$ for $t \geq t_{k}$ (and 0 for $t \leq t_{k}$ ) leading to an overall time-dependent signal $A(t)=\sum_{k} A\left(t, t_{k}\right)$. The power spectrum of the fluctuating part of this signal is ${ }^{22}$

$$
S(f)=\frac{A_{0}^{2} n}{\lambda^{2}+(2 \pi f)^{2}}
$$

which is frequently referred to as Debye relaxation. In the limit $f \gg \lambda$, the Debye term approaches a simple $1 / f^{2}$ behaviour.

If there are multiple time-scales, i.e. if the relaxation frequency of each of the single events is drawn from a probability distribution, $g_{\lambda}(\lambda)$, then the power spectrum must be computed as:

$$
S(f)=\frac{n\left\langle A^{2}\right\rangle}{2 \pi} \int_{\lambda_{\min }}^{\lambda_{\max }} \frac{g_{\lambda}(\lambda)}{(2 \pi f)^{2}+\lambda^{2}} d \lambda
$$


If $g_{\lambda}(\lambda)$ corresponds to a uniform distribution over the range $\lambda_{1}$ to $\lambda_{2}$ and the amplitude of each pulse remains constant, then

$$
\begin{aligned}
S(f) & =\frac{1}{\lambda_{2}-\lambda_{1}} \int_{\lambda_{1}}^{\lambda_{2}} \frac{A_{0}^{2} n}{\lambda^{2}+(2 \pi f)^{2}} d \lambda \\
& =\frac{A_{0}^{2} n}{2 \pi f\left(\lambda_{2}-\lambda_{1}\right)}\left[\arctan \left(\lambda_{2} / 2 \pi f\right)-\arctan \left(\lambda_{1} / 2 \pi f\right)\right]
\end{aligned}
$$

In the low-frequency limit such that $f \ll \lambda_{1} \ll \lambda_{2}$, the spectrum flattens and we observe white noise with $\langle S(f)\rangle=A_{0}^{2} n$. In the intermediate region where $\lambda_{1} \ll f \ll \lambda_{2}$, we obtain

$$
S(f)=\frac{A_{0}^{2} n}{4 f\left(\lambda_{2}-\lambda_{1}\right)}
$$

which corresponds to the $1 / f^{\alpha}$ regime with $\alpha=1$. In the high-frequency limit for which $f \gg \lambda_{2} \gg \lambda_{1}$, we obtain a $1 / f^{2}$ noise with $S(f)=A_{0}^{2} n /(2 \pi f)^{2}$. Other distributions of time scales may also give rise to $1 / f^{\alpha}$ behaviour; for example, a distribution $P(\lambda) \propto \lambda^{-\beta}$ over the same frequency interval will generate $S(f) \propto 1 / f^{1+\beta}$. Ref $\underline{\underline{34}}^{\frac{34}{4}}$ provides a gallery of spectral densities associated with different models. In general, a spectral index $\alpha$ in the interval $0.5<\alpha<1.8$ may be taken as an indication of multiple time-scale dynamics while exponents very close to 2 indicate single time-scale behaviour. A separation of two decades in time scales between $\lambda_{1}$ and $\lambda_{2}$ is sufficient to observe a $1 / f^{\alpha}$ regime with $\alpha$ close to 1 . Unless otherwise stated, in this work, reference to $1 / f^{\alpha}$ behaviour will imply the multiple time-scale regime with $\alpha$ between 0.5 and 1.8 , rather than the Debye tail with $\alpha \approx 2$.

In the case of a networked liquid, like water, the multiple time scale region spans approximately two decades. There may be additional features, such as high-frequency resonances or low-frequency Debye relations. One can, in principle, disentangle the different contributions to the complex network dynamics using a model-based fitting procedure ${ }^{34}$. For the purpose of quantitatively characterising the changes in the hydrogen bond network dynamics in the diffusionally anomalous region, we have found it sufficient to identify the $1 / f^{\alpha}$ region from the $\ln S(f)$ versus $\ln f$ plot and obtain the scaling exponent $\alpha$ by numerical fitting.

\section{COMPUTATIONAL DETAILS}

\section{A. Potential Model}

We use the van Beest-Kramer-van Santen (BKS) potential to model interatomic interactions in silica since it has been extensively used to study liquid state anomalies and the glass 
transition ${ }^{23,24}$. The BKS potential is pair-additive and contains long-range Coulombic and short-range two-body contributions. The pair interaction between atoms $i$ and $j$ is given by:

$$
\phi_{B K S}\left(r_{i j}\right)=\frac{q_{i} q_{j}}{4 \pi \varepsilon_{0} r_{i j}}+A_{i j} \exp ^{-b_{i j} r_{i j}}-\frac{C_{i j}}{r_{i j}^{6}}
$$

where $r_{i j}$ is the distance between atoms $i$ and $j$ carrying charges $q_{i}$ and $q_{j}$ and $A_{i j}, b_{i j}$ and $C_{i j}$ are the parameters associated with the Buckingham potential for short-range repulsiondispersion interactions. In the original BKS potential, the Buckingham parameters for the Si-Si interactions are taken as zero. At high temperatures and pressures, the BKS potential exhibits an unphysical divergences in the interaction energy when the Si-O distance becomes very small. To remove these divergences, a short range correction term was added by Poole et. $\mathrm{al}^{26}$ to the BKS potential and the parameters adjusted in such a way that the original form was left unchanged at larger separations while the negative divergence was avoided at smaller separations. The modified pair interaction is

$$
\phi\left(r_{i j}\right)=\phi_{B K S}+4 \epsilon_{i j}\left[\left(\frac{\sigma_{i j}}{r_{i j}}\right)^{30}-\left(\frac{\sigma_{i j}}{r_{i j}}\right)^{6}\right]
$$

where $\epsilon_{i j}$ and $\sigma_{i j}$ are the energy and length scale parameters for the 30-6 Lennard-Jones interaction. The parameters for the modified BKS potential used in this work are given in Table I.

\section{B. Molecular Dynamics}

Molecular Dynamics simulations of a system of $150 \mathrm{Si}$ and $300 \mathrm{O}$ ions were carried out in canonical $(N-V-T)$ ensemble, using the DL_POLY software package ${ }^{32.33}$, under cubic periodic boundary conditions. The effects of electrostatic (long-range) interactions were accounted for by the Ewald summation method ${ }^{30,31}$. The non-coulombic part was truncated and shifted at $7.5 \AA$. A Berendsen thermostat, with the time constant $\tau_{B}=200 \mathrm{ps}$, was used to maintain the desired temperature for the production run. The Verlet algorithm with a time step of $1 \mathrm{fs}$ was used to integrate the equations of motion. The system was simulated at 5 temperatures in the $4000 \mathrm{~K}$ to $6000 \mathrm{~K}$ range lying along 8 isochores in the density range from $1.8 \mathrm{~g} \mathrm{~cm}^{-3}$ to $4.2 \mathrm{~g} \mathrm{~cm}^{-3}$. For temperatures above $5000 \mathrm{~K}$, the system was equilibrated for $3-4 \mathrm{~ns}$ followed by a production run of 5-7ns. For lower temperatures, an equilibration period of 5-6ns was followed by a production run of 8-10ns. The starting configuration for the liquid state simulations was generated by replicating the cubic unit cell of $\beta$-cristobalite to generate a 
cube of edge length $21.48 \AA^{37.38}$ which was then suitably truncated to obtain a simulation cell containing $150 \mathrm{Si}$ and 300 oxygen atoms. The system thus obtained was heated gradually to form the equilibriated liquid state. Our simulation results are in agreement with previous results ${ }^{10,26}$ within the statistical error bars.

Figure[1] shows the $P(\rho)$ curve at different temperatures. It can be seen that at all densities below $3.0 \mathrm{~g} \mathrm{~cm}^{-3}$, the pressures are negative for the temperatures studied, indicating that liquid silica is stretched rather than compressed. The isothermal compressibility, defined as $\kappa_{T}=(1 / \rho)(\partial \rho / \partial P)$, is negative only for the $\rho=1.8 \mathrm{~g} \mathrm{~cm}^{-3}$ isochore. The spindonal density for which $\kappa_{T}=0$ is located around $2.0 \mathrm{~g} \mathrm{~cm}^{-3}$. Below this density, the system is likely to be inhomogenous. In our simulations, the state points along the $1.8 \mathrm{~g} \mathrm{~cm}^{-3}$ isochore are thermodynamically unstable; however, to present a complete study to explore the diffusional anomaly we consider this density.

\section{Power spectral analysis}

In this study, we focus exclusively on the power spectra associated with tagged atom potential quantities, $u_{O}$ and $u_{S i}$, which were stored at intervals of $10 \mathrm{fs}$ during the MD runs. We represent power spectra of tagged potential energy fluctutations as $S_{u}(f)$ but while referring to $S_{u}(f)$ spectra of atoms of a specific type A, we use the notation $S_{A}(f)$. The sampling interval of $10 \mathrm{fs}$ corresponds to a Nyquist frequency of $1666 \mathrm{~cm}^{-1}$. The value of the Berendsen thermostat time constant $\left(\tau_{B}\right)$ provides the lower limit on the frequency range over which we can obtain reliable power spectra; thus, $\tau_{B}=200 \mathrm{ps}$ corresponds to a lower frequency limit of $0.165 \mathrm{~cm}^{-1}$. Standard Fast Fourier Transform routines were used with a square sampling window $\underline{20}$. The normalisation convention was chosen such that the integrated area under the $S(f)$ curve equalled the mean square amplitude of the time signal. Windows containing $2^{19}$ data points were used for Fourier transformation. Statistical noise in the power spectra was reduced by averaging over overlapping time signal windows as well as over individual tagged particle spectra. In a given frequency interval showing $1 / f^{\alpha}$ behaviour, linear least squares fitting of $\ln S(f)$ was done to obtain the $\alpha$ values. 


\section{RESULTS}

Figure 2 shows the behaviour of the self-diffusivities, $D_{S i}$ and $D_{O}$ of $\mathrm{Si}$ and $\mathrm{O}$ atoms respectively, in BKS silica melt as a function of density along different isotherms. At any given state point, the oxygen self-diffusivities are greater than those of silicon atoms by approximately a factor of two but the density and temperature-dependent trends are very similar. Subsequently, in this paper, we will focus only on $D_{S i}$ since silicon atoms are the sites with the local tetrahedral symmetry. The diffusionally anomalous region is clearly demarcated in the isotherms lying between $4000 \mathrm{~K}$ and $5000 \mathrm{~K}$. The density of minimum diffusivity, $\rho_{\text {min }}$, is approximately $2.0 \mathrm{~g} \mathrm{~cm}^{-3}$ over this temperature range while the density of maximum diffusivity, $\rho_{\max }$, is approximately $3.4 \mathrm{~g} \mathrm{~cm}^{-3}$. In the case of SPC/E water, the region of anomalous diffusivity is seen below $280 \mathrm{~K}$ and the $\rho_{\min }$ and $\rho_{\max }$ values are approximately $0.9 \mathrm{~g} \mathrm{~cm}^{-3}$ and $1.1 \mathrm{~g} \mathrm{~cm}^{-3}$ respectively.

Figure 3 compares the power spectra generated by fluctuations in potential energies of tagged oxygen and silicon atoms in silica melt at $4000 \mathrm{~K}$ along the $2.0 \mathrm{~g} \mathrm{~cm}^{-3}$ isochore with that of water at $0.9 \mathrm{~g} \mathrm{~cm}^{-3}$ at $230 \mathrm{~K}$. The chosen state point for both systems corresponds to the density minimum along the appropriate isotherm. Despite the difference in intermolecular interactions between a molecular liquid and an ionic melt, there is a striking parallel in the power spectral features suggesting a very similar pattern in the dynamical correlations.

The $S_{S i}(f)$ spectrum shows a sharp peak at $1100 \mathrm{~cm}^{-1}$ corresponding to the to the SiO-Si asymmetric stretch vibration seen in both the experimental as well as simulated IR spectrum 35.36 . The power spectrum also shows a secondary, less intense peak at $800 \mathrm{~cm}^{-1}$ corresponding to $\mathrm{O}-\mathrm{Si}-\mathrm{O}$ bending. It should be noted that the tagged particle power spectrum reflects the intrinsic dynamics of the liquid state network while the infra-red spectrum highlights those modes which couple to the electric field of the electromagnetic radiation in the IR frequency range. The localised high frequency peaks at 1100 and $800 \mathrm{~cm}^{-1}$ correspond to well-defined local vibrational modes of the $\mathrm{SiO}_{4}$ tetrahedra and mirror the librational peak in water which is, however, somewhat broader and more intense.

The region between 0.2 and $400 \mathrm{~cm}^{-1}$ in $S_{S i}(f)$ may be regarded as essentially a multiple time scale or $1 / f^{\alpha}$ region with a frequency-dependent value of $\alpha$. At higher temperatures or densities, the very low frequency region shows a cross-over to white noise. The multiple time scale region of water spans a similar frequency range.

The $S_{S i}(f)$ and $S_{O}(f)$ spectra are qualitatively very similar. The tagged oxygen power spectrum, $S_{O}(f)$, typically has a lower intensity than the $S_{S i}(f)$ power spectrum because 
fluctuations in the tagged energy of the oxygen atoms are significantly lower than those for the $\mathrm{Si}$ atoms due to the relative magnitude of the charges. The high-frequency peaks in the $S_{O}(f)$ spectra occur at essentially the same locations as in $S_{S i}(f)$ but have significantly different intensities suggesting that tagged energies of the two types of atoms have different sensitivities to the local vibrational modes.

Section 2 discusses the different contributions to the tagged atom potential energy i.e. van der Waals $\left(u_{v d w}\right)$, screened charge $\left(u_{\text {screen }}\right)$, self-interaction $\left(u_{\text {self }}\right)$ and reciprocal space $\left(u_{r e c}\right)$. While $u_{\text {self }}$ is a constant for a given atomic type, the power spectra associated with fluctuations in the other three quantities at the state point $\left(\mathrm{T}=4000 \mathrm{~K}, 2.0 \mathrm{~g} \mathrm{~cm}^{-3}\right)$ is shown in Figure 4. The behaviour of $S_{u}(f)$ is almost exactly reproduced over much of the frequency range by the screened charge contribution. The spectral power associated with the van der Waals and reciprocal space contributions is much lower and therefore has a minor effect on the overall spectrum except in the high-frequency region. Unlike in the case of water, the $u_{v d w}$ contribution produces a relatively unstructured power spectrum, specially in the case of oxygen, while the Coulombic interactions are responsible for much of the high-frequency, short length scale structure. This is corroborated by the static distributions of the various contributions to the tagged particle potential energies shown in Figure 5. The width of the $P\left(u_{r e c}\right)$ and $P\left(u_{v d w}\right)$ distributions is much narrower than that of $P\left(u_{\text {screen }}\right)$ both in the case of $\mathrm{Si}$ and $\mathrm{O}$ atoms.

To understand the effect of pressure and temperature on the tetrahedral network in liquid silica, we examined the power spectra as well as static distributions of tagged particle potential energies as a function of temperature and pressure. Figures 6 and 7 show the results along the $2.0 \mathrm{~g} \mathrm{~cm}^{-3}$ isochore and the $4000 \mathrm{~K}$ isotherm.

Figure 6(a) shows that, along a given isochore, the crossover to white noise occurs at increasingly higher frequencies as temperature increases. Moreover the local vibrational peak broadens and the upper bound of the frequency range for multiple time-scale or $1 / f^{\alpha}$ behaviour increases. This progressive coupling of local modes into network vibrations leads to a change in the exponent $\alpha$ of the $1 / f^{\alpha}$ region. At the lowest temperatures, the frequency dependence of $\alpha$ is more pronounced. For example, at $4000 \mathrm{~K}$ and $2.0 \mathrm{~g} \mathrm{~cm}^{-3}$ one can identify a high frequency regime from $20-200 \mathrm{~cm}^{-1}$ with $\alpha=0.8029$ while between $1-20 \mathrm{~cm}^{-1}$, the $\alpha$ value is 1.6526. Since we wish to use $\alpha$ as a measure of the degree of coupling of local and network modes, we consider $\alpha$ in the $20-200 \mathrm{~cm}^{-1}$ region. As we show below, this change is quantitatively correlated with increasing frequency in the anomalous region. 
The effect of increasing density or pressure on the power spectrum is shown in Figure 6(b). The crossover to white noise occurs at about $1 \mathrm{~cm}^{-1}$ for densities of $3.0 \mathrm{~g} \mathrm{~cm}^{-3}$ or more. The effect of increasing pressure results in loss of the bimodal character of the highfrequency peak. The peak at approximately $800 \mathrm{~cm}^{-1}$ corresponding to the O-Si-O bend is clearly much more sensitive to pressure than the Si-O-Si asymmetric stretch; this is not surprising since it is known that compaction results in distortion of bond angles resulting in more efficient packing of $\mathrm{SiO}_{4}$ tetrahedra. This is different from the loss of the distinct identity of the librational peak seen in water with increasing density or temperature.

Figure 7 shows the static distributions, $P(u)$ of the tagged atom potential energies. At low temperatures or low densities, the $P\left(u_{S i}\right)$ distributions are multimodal with six distinct peaks. This suggests that the energetic environment of a given $\mathrm{Si}$ atom depends on bond lengths and bond angles associated with the nearest four oxygen atoms forming the local $\mathrm{SiO}_{4}$ tetrahedral unit. When all the angles and bond lengths are optimal, the lowest energy environment is obtained. The presence of distinct peaks suggests that there is a small energetic barrier to transit from one energetic environment to another. The effect of temperature is to allow for more facile transitions between different energetic environments which results in a loss of multimodality but no additional broadening of the distributions. In contrast, the effect of pressure is to both broaden as well as destructure the distributions. The $P\left(u_{O}\right)$ has two major and one minor peak at low temperatures or low densities. With increasing temperature, the minor peak becomes part of the shoulder of the low energy peak. With increasing density, the minor, central peak disappears and the overall wiidth of the distribution broadens.

The earlier study on water has established the strong correlation between the diffusivity and the scaling exponent $\alpha$ of the multiple time-scale regime in the anomalous regime. Figures 8 and 9 show that this relationship also holds in the case of liquid silica. Figure 8 shows the behaviour of the scaling exponent $\alpha_{u}^{S i}$ of the $S_{S i}(f)$ spectra as a function of density along different isotherms. The parallel with the diffusivity plot in Figure 2 is obvious. It should be noted, however, that $\alpha$ is essentially constant at 1.5 at high temperatures and densities when the liquid is in the diffusionally normal regime. Figure 9 shows a correlation plot of $\alpha_{u}^{S i}$ with $\ln D_{S i}$ where the clustering of $\alpha$ values around 1.5 can be seen. 


\section{DISCUSSION AND CONCLUSIONS}

This study demonstrates the strong parallels in the dynamical behaviour of the liquid state networks in silica and water in the anomalous regime. Power spectral analysis is a convenient tool for characterising the complex dynamics of network-forming liquids, such as silica, since the multiple time-scale behaviour of the network is captured by the range and exponent of the $1 / f^{\alpha}$ regime. It is particularly useful for understanding diffusion in the random network of the liquid. In normal liquids, diffusion proceeds via collisions and relaxations of the local nearest-neighbour cage. In networked liquids, local vibrational modes can couple to network reorganisations, thereby facilitating diffusion. The details of the process in water and silica differ, but in both cases, the degree of coupling can be indexed by the exponent $\alpha$ of the multiple time-scale regime. As a consequence, the diffusivity is strongly correlated with the scaling exponent, specially in the anomalous regime. As the network connectivity is attenuated by temperature and pressure in the normal regime, the correlation begins to break down.

The results of this study provide some insights into the microscopic connection between the structural and diffusional anomalies. The boundaries of the structurally anomalous region are determined by extrema in the local order parameters. The progressive destruction of local tetrahedral order by temperature or pressure has an effect on the local atomic or molecular environment, as can be seen from the static distribution of the tagged particle potential energies. As the local tetrahedral order is destroyed, the vibrational coupling of local modes, specially the O-Si-O bend, to the network reorganisations becomes much stronger causing a rise in diffusivity. The diffusivity does not, however, rise indefinitely with increasing compression because for sufficiently large values of the density, the extent and connectivity of the network is severely attenuated and the liquid shifts from the anomalous to the normal regime. Therefore one would expect the diffusional and structural anomalies to be strongly correlated. Whether the structural anomalies precede or succeed the diffusional anomaly (as in water and silica respectively) must depend on the details of the potential energy surface, such as the nature and extent of vibrational coupling. Based on the similarity in power spectral behaviour of SPC/E and TIP5P-E models for water ${ }^{19}$, we would expect different effective pair potentials for silica to have very similar qualitative behaviour. It is possible, however, that three-body potentials for silica will show more significant deviations, such as a reversal in the relative order of the two types of anomalies ${ }^{35}$.

The parallel behaviour of power spectra of water and silica in the diffusionally anomalous 
region suggests that all tetrahedral liquids should display this generic pattern of behaviour. It would be interesting to perform a power spectral analysis of other liquids showing waterlike anomalies, such as two-scale ramp potentials ${ }^{39}$, to see if the detailed dynamical behaviour resembles that of tetrahedral liquids.

Acknowledgements This work was supported by the Department of Science and Technology, New Delhi, under the Swarnajayanti Fellowship scheme. RS thanks Council for Scientific and Industrial Research, New Delhi, for the award of a Junior Research Fellowship. 
1 Theory of Simple Liquids edited by J. P. Hansen and I. R. McDonald, (Academic Press, London, 1986).

2 P. G. Debenedetti, J. Phys.: Condensed Matter 2003, 15 (R1669).

3 Hydration Processes in Biology edited by M.-C. Belissent-Funel (Ed.) (IOS Press, Amsterdam, 1999).

4 C. A. Angell and H. Kanno, Science 193, 1121 (1976).

5 C. A. Angell, R. D. Bressel, M. Hemmati, E. J. Sare and J. C. Tucker, Phys. Chem. Chem. Phys. 2, 1559 (2000).

6 M. H. Ford, S. M. Auerbach and P. A. Monson, J. Chem. Phys. 121, 8415 (2004).

7 H. Tanaka, Phys. Rev. B 66, 064202 (2002).

8 C. J. Roberts and P. G. Debendetti, J. Chem. Phys. 105, 658 (1996).

9 J. R. Errington and P. G. Debenedetti, Nature 409, 318 (2001).

10 M. S. Shell, P. G. Debendetti and A. Z. Panagiotopoulos, Phys. Rev. E. 66, 011202 (2002).

11 J. R. Errington, P. G. Debenedetti and S. Torquato, Phys. Rev. Lett. 89, 215503 (2002).

12 J. R. Errington, P. G. Debenedetti and S. Torquato, J. Chem. Phys. 118, 2256 (2003).

13 P. Raiteri, A. Laio and M. Parrinello, Phys. Rev. Lett. 93, 087801 (2004).

14 A. Mudi, R. Ramaswamy and C. Chakravarty, Chem. Phys. Lett. 376, 683 (2003).

15 A. Mudi and C. Chakravarty, Mol. Phys. 102, 681 (2004).

16 A. Mudi and C. Chakravarty, J. Phys. Chem. B 108, 19607 (2004).; ibid. 110, 4502 (2006).

17 A. Mudi, C. Chakravarty and R. Ramaswamy, J. Chem. Phys. 122, 104507 (2005).; ibid. 124, $069902(2006)$.

18 A. Mudi and C. Chakravarty, J. Phys. Chem. B 110, 8422 (2006).

19 A. Mudi, C. Chakravarty and E. Milotti, J. Chem. Phys. (to be published).

20 W. H. Press, B. P. Flannery, S. A. Teukolsky, and W. T. Vetterling Numerical Recipes in FORTRAN (Cambridge University Press, Cambridge, 1990.)

21 B. Mandelbrot, Multifractals and 1/f noise: Wild self-affinity in Physics (1963-1976) (SpringerVerlag, New York, 1999).

22 E. Milotti, 1/f noise: a pedagogical review http://lanl.arXiv.org/physics/0204033

23 B. W. H van Beest, G. J. Kramer and R. A. van Santen, Phys. Rev. Lett. 64, 1955 (1990). 
24 G. J. Kramer, N. P. Farragher and B. W. H van Beest Phys. Rev. B 43, 5068 (1991).

25 I. Saika-Voivod, F. Sciortino, T. Grande and P. H. Poole, Phys. Rev. E. 70, 061507 (2004).

26 I. Saika-Voivod, F. Sciortino and P. H. Poole, Phys. Rev. E. 63, 011202 (2000).

27 I. Saika-Voivod, P. H. Poole and F. Sciortino, Nature 412, 514 (2001).

28 E. La Nave, H. E. Stanley and F. Sciortino, Phys. Rev. Lett. 88, 035501 (2002).

29 M. Hemmati and C. A. Angell, J. Non-Cryst. Soilds 217, 236 (1997).

30 M. D. Allen and D. J. Tildesley, Computer Simulation of Liquids (Clarendon Press, Oxford, 1986).

31 D. Frenkel, and B. Smit Understanding Molecular Simulation: From Algorithms to Applications; Academic Press: London, 2002.

32 W. Smith and T. R. Forester, J. Mol. Graphics 14 (1996) 136.

33 W. Smith, C. W. Yong and P. M. Rodger, Mol. Simulat. 28, 385 (2002).; The DL_POLY website is http://www.cse.clrc.ac.uk/msi/software/DL_POLY/

34 E. Milotti, Model-based fit procedure for power-law-like spectra, J. Comp. Phys. (in press); also: arXiv:physics/0510011.

35 L. Huang and J. Kieffer, J. Chem. Phys. 118, 1487 (2003).

36 A. G. Kalampounias, S. N. Yannopoulos and G. N. Papatheodorou, J. Chem. Phys. 124, , (0).14504 (2006).

37 P. Villars and L. D. Calvert, Pearson's Handbook of Crystallographic Data for Intermetallic Phases, $2^{\text {nd }}$ Edition (ASM International, Materials Park, Ohio, 1991). Also see : http://cst-www.nrl.navy.mil/lattice/struck/c9.html

38 R. W. G. Wyckoff, Crystal Structures, Volume 1, p - 318-19, (John Wiley and Sons, New York, London, 1963)

39 Z. Yan, S. V. Buldyrev, N. Giovambattista and H. E. Stanley. Phys. Rev. Lett. 95, 130604 (2005). 


\section{Table I}

BKS (modified) potential parameters ${ }^{23,24}$.

\begin{tabular}{|c|c|c|c|c|c|}
\hline$i-j$ & $\begin{array}{c}A_{i j} \\
\left(\mathrm{~kJ} \mathrm{~mol}^{-1}\right)\end{array}$ & $\begin{array}{c}b_{i j} \\
(\stackrel{\stackrel{A}{A}}{-1})\end{array}$ & $\begin{array}{c}C_{i j} \\
\left(\mathrm{~kJ} \mathrm{~mol}^{-1}\right)\end{array}$ & $\begin{array}{c}\epsilon_{i j} \\
\left(\mathrm{~kJ} \mathrm{~mol}{ }^{-1}\right)\end{array}$ & $\begin{array}{l}\sigma_{i j} \\
(\stackrel{\circ}{A})\end{array}$ \\
\hline$O-O$ & 134015 & 2.76 & 16887.3 & 0.101425 & 1.7792 \\
\hline$S i-O$ & 1737340 & 4.87 & 12886.3 & 0.298949 & 1.3136 \\
\hline
\end{tabular}




\section{Figure Captions}

1. Equation of state for BKS silica: Pressure $(P)$ as a function of density $(\rho)$ along different isotherms. Systems at a density of $1.8 \mathrm{~g} \mathrm{~cm}^{-3}$ are likely to be inhomogeneous.

2. Dependence on density, $\rho$, of (a) self-diffusivity of $\mathrm{Si}, D_{S i}$, and (b) self-diffusivity of $\mathrm{O}, D_{O}$ along different isotherms.

3. Comparison of power spectra associated with temporal fluctuations in tagged particle potential energy of $\mathrm{Si}$ abd $\mathrm{O}$ atoms at $4000 \mathrm{~K}$ and $2.0 \mathrm{~g} \mathrm{~cm}^{-3}$ with water at $230 \mathrm{~K}$ and $0.9 \mathrm{~g} \mathrm{~cm}^{-3}$. The power spectrum of $\mathrm{O}$ atoms has been scaled to half for clarity.

4. Contributions of reciprocal space and screening from Ewald summation and van der Waals interactions to the power spectra of total tagged potential energy fluctuations of (a) Si at $4000 \mathrm{~K}$ and $2.0 \mathrm{~g} \mathrm{~cm}^{-3}$ and (b) $\mathrm{O}$ at $4000 \mathrm{~K}$ and $2.0 \mathrm{~g} \mathrm{~cm}^{-3}$. Relative magnitudes kept unaltered.

5. Contributions of reciprocal space and screening from ewald summation and van der Waals interactions to the static distribution of tagged particle potential energy of (a) Si at $4000 \mathrm{~K}$ and $2.0 \mathrm{~g} \mathrm{~cm}^{-3}$ and (b) $\mathrm{O}$ at $4000 \mathrm{~K}$ and $2.0 \mathrm{~g} \mathrm{~cm}^{-3}$. In part (a) van der Waals and reciprocal space contributions, $\mathrm{P}\left(u_{v d w}\right)$ and $\mathrm{P}\left(u_{r e c}\right)$ respectively, have been scaled by a factor of $1 / 10$. In part (b) $\mathrm{P}\left(u_{v d w}\right)$ has been scaled by $1 / 4$ while $\mathrm{P}\left(u_{\text {rec }}\right)$ has been scaled by $1 / 10$.

6. Power spectra of tagged particle potential energy fluctuations, $S_{u}(f)$, of Si in liquid silica along (a) $2.0 \mathrm{~g} \mathrm{~cm}^{-3}$ isochore from $4000 \mathrm{~K}$ to $6000 \mathrm{~K}$ in steps of $500 \mathrm{~K}$ and (b) $4000 \mathrm{~K}$ isotherm at 1.8, 2.0, 2.2, 2.6, 3.0, 3.4, 3.8 amd $4.2 \mathrm{~g} \mathrm{~cm}^{-3}$. Relative magnitudes of power spectra for different temperatures and densities were adjusted (scaled) for clarity.

7. Static distributions of tagged particle potential energies (in $\mathrm{kJ} \mathrm{mol}^{-1}$ ) of (a) Si along $2.0 \mathrm{~g} \mathrm{~cm}^{-3}$ isochore, (b) Si along $4000 \mathrm{~K}$ isotherm, (c) $\mathrm{O}$ along $2.0 \mathrm{~g} \mathrm{~cm}^{-3}$ isochore and (d) O along 4000K isotherm.

8. Multiple time-scale exponent, $\alpha_{u}^{S i}$, as a function of density along different isotherms. The exponent $\alpha_{u}^{S i}$ is evaluated using the high frequency $1 / f^{\alpha}$ regime of $20-200 \mathrm{~cm}^{-1}$ at $4000 \mathrm{~K}$ and $4500 \mathrm{~K}$ and from the point of crossover to $200 \mathrm{~cm}^{-1}$ for $5000 \mathrm{~K}, 5500 \mathrm{~K}$ and $6000 \mathrm{~K}$. 
9. Correlation plot showing $\ln D_{S i}$ against $\alpha_{u}^{S i}$ for different isochores. The frequency range for evaluating $\alpha_{u}^{S i}$ are the same as in Figure 8. Units of diffusivity are taken as $10^{-5} \mathrm{~cm}^{2} \mathrm{~s}^{-1}$. 


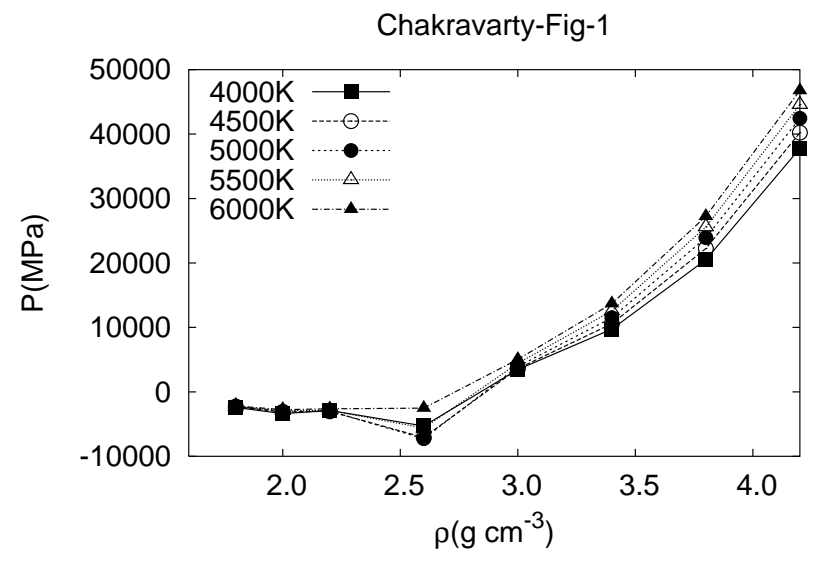



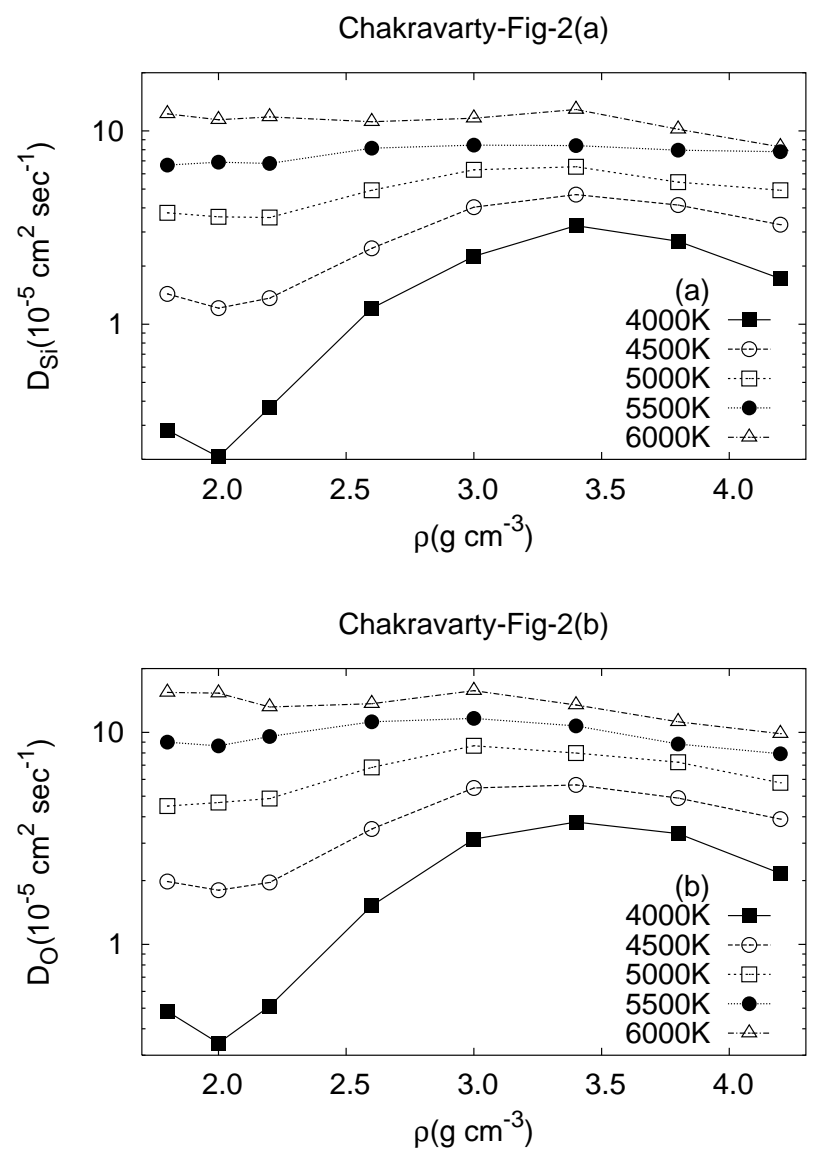


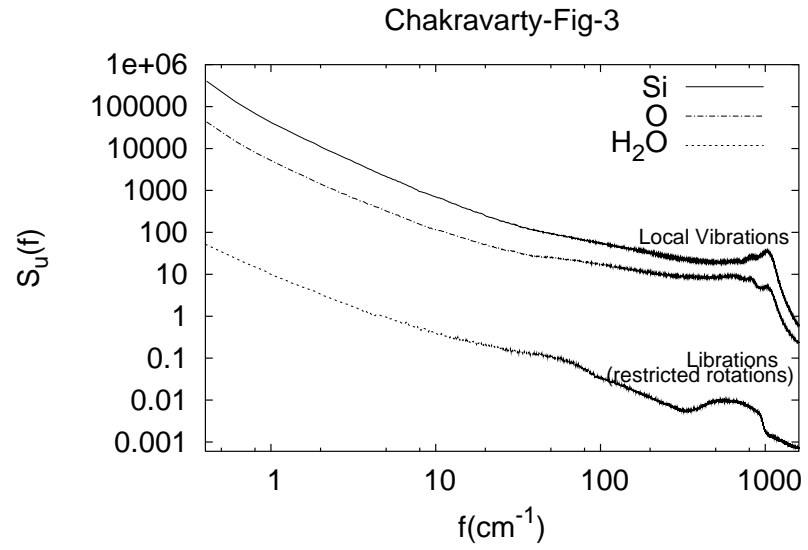



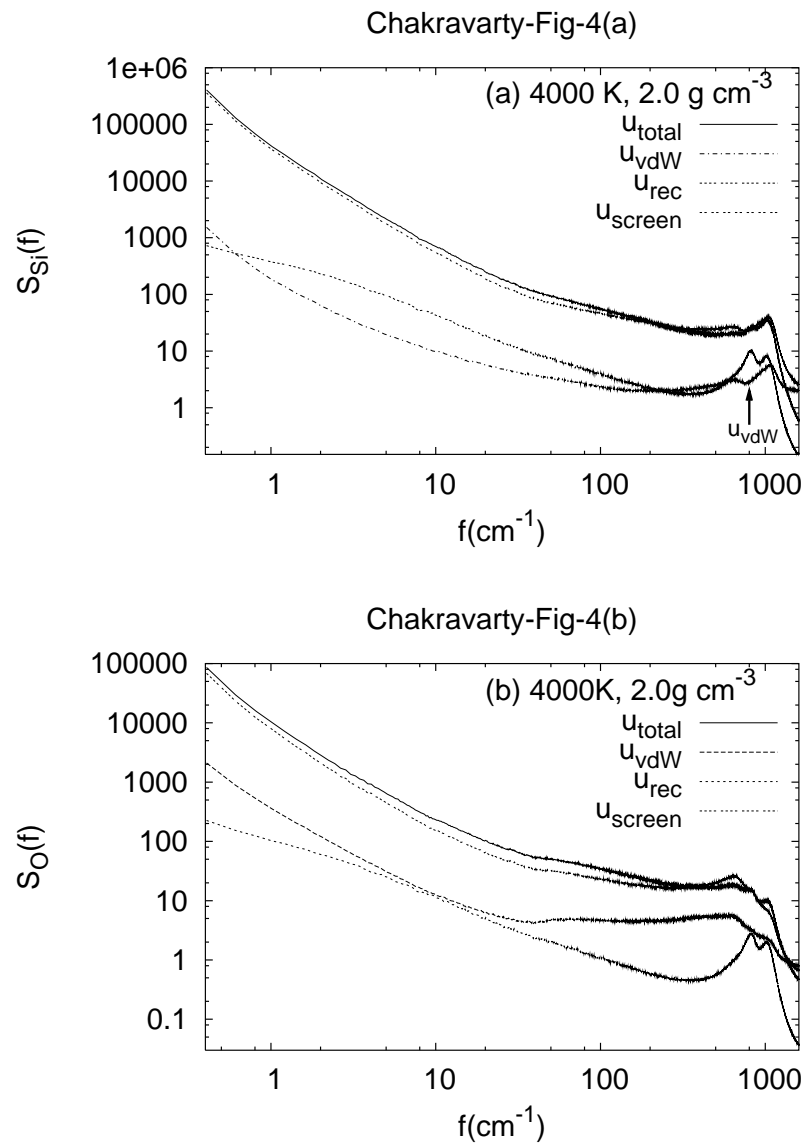

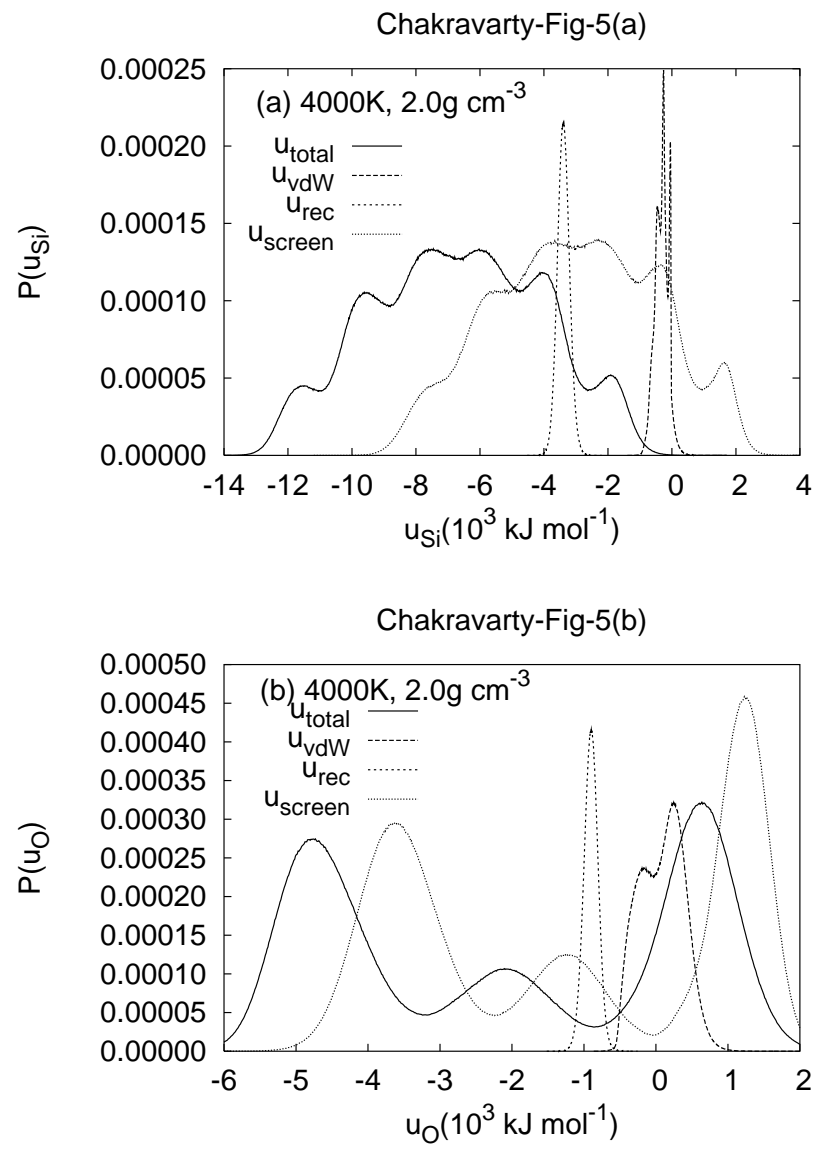

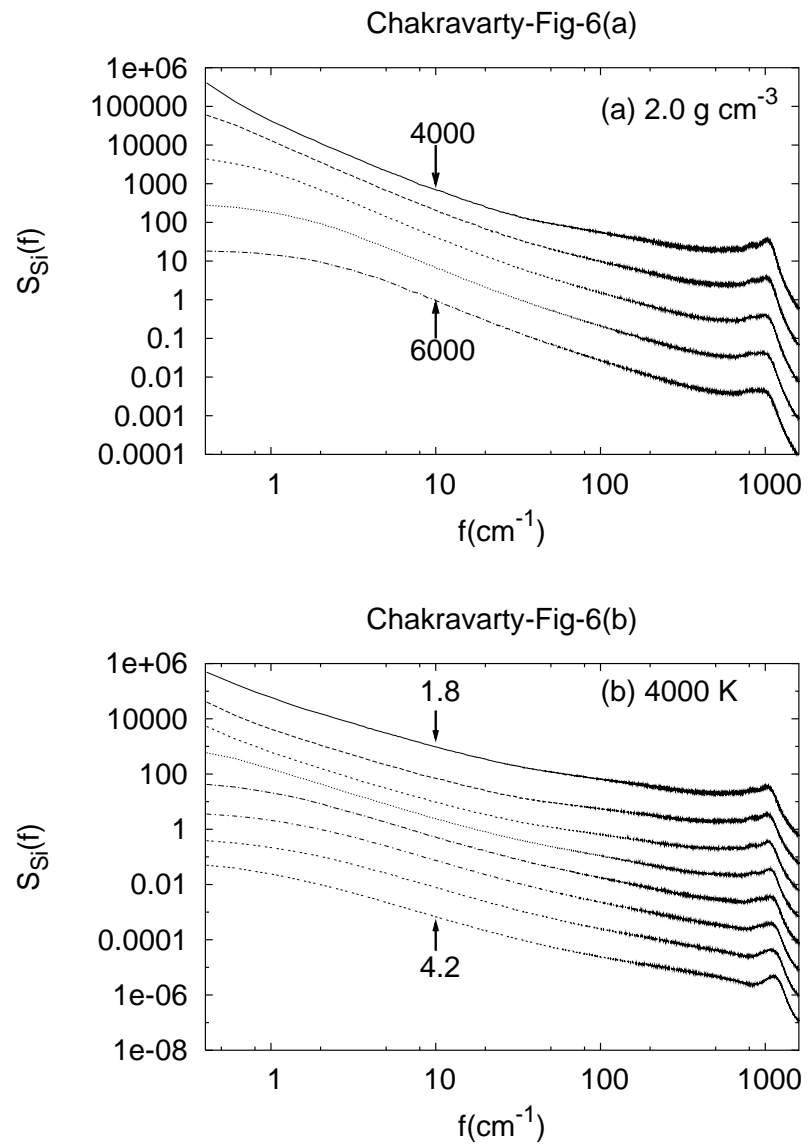

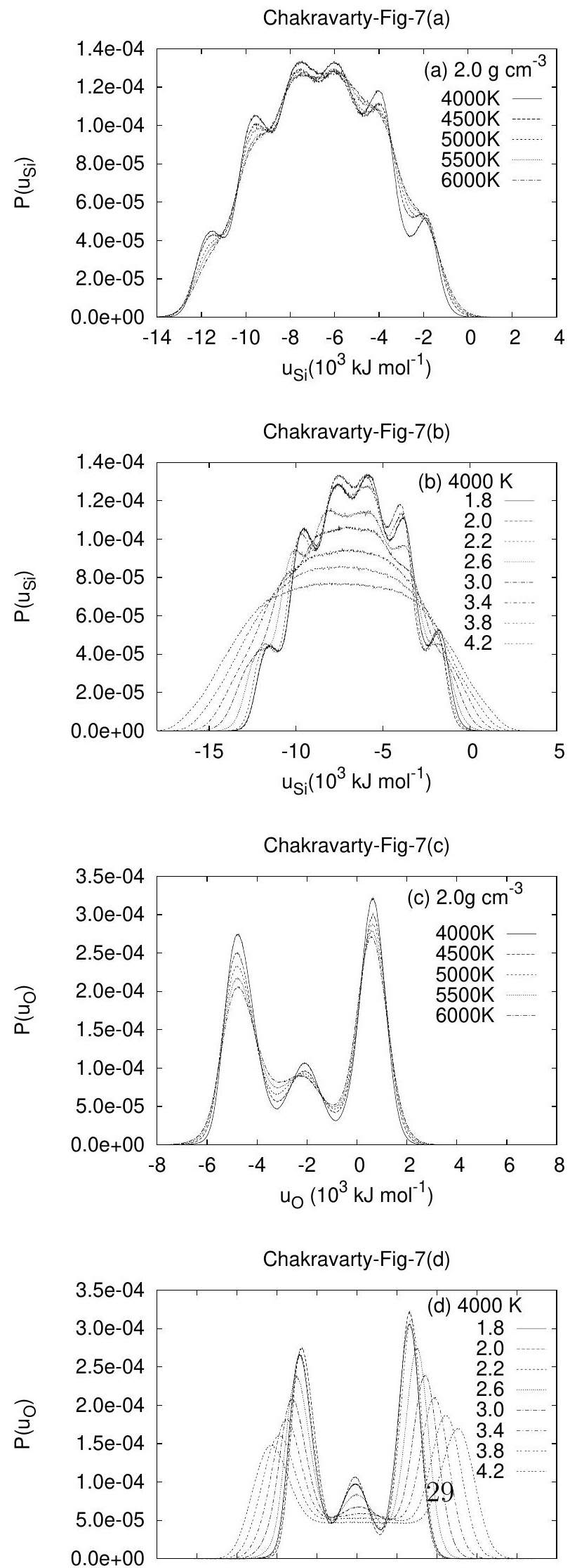


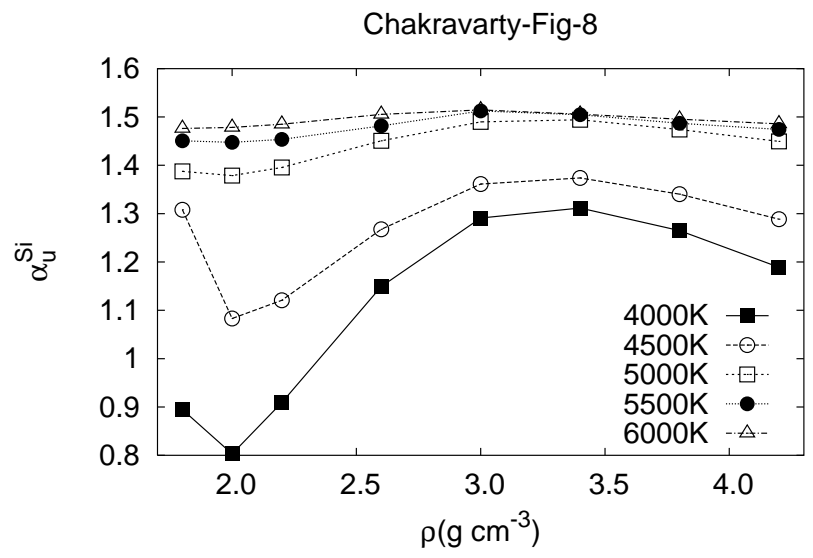




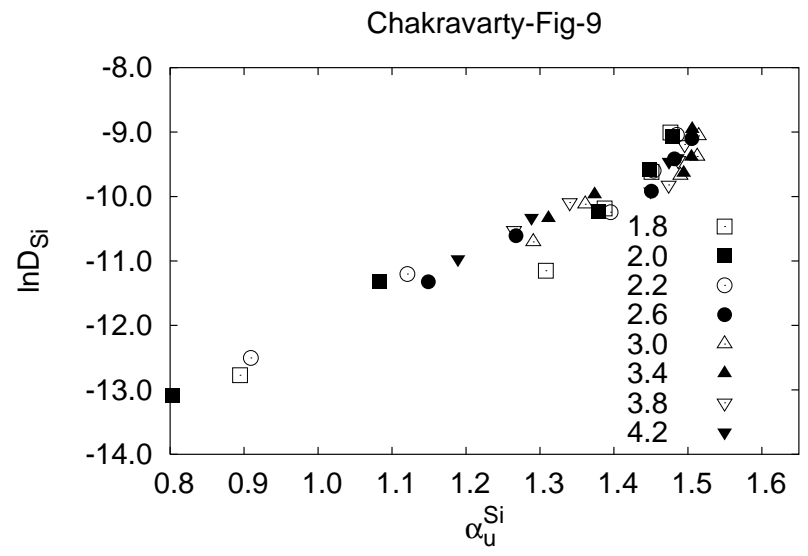

